\title{
BRCA germline mutation test for all woman with ovarian cancer?
}

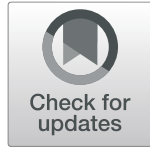

\author{
A. V. Paradiso ${ }^{1,4^{*}}$, M. Digennaro ${ }^{1}$, M. Patruno ${ }^{1}$, S. De Summa ${ }^{2}$, S. Tommasi ${ }^{2}$ and I. Berindan-Neagoe ${ }^{3}$
}

\begin{abstract}
Background: Delivering widespread BRCA testing to patients with ovarian cancer has been suggested by several scientists, recommended by professional societies and solicited by patients organizations. However, based on the lack of studies clearly demonstrating the cost-effectiveness of such approach compared to standard practice, we evaluated the possibility to better select subgroups of ovarian cancer (OC) patients with higher probability to be a BRCA mutation carrier'.

Methods: We analyzed the database of 2222 germline BRCA analyses from OC patients recently published by Song et al. (Song 2014) by applying multivariate and conditional inference regression tree-analyses.

Results: Overall, 178/2192 (8.1\%) evaluable OC women showed pathogenic germline mutations in BRCA genes (84 BRCA1;94 BRCA2). BRCA mutations resulted significantly more frequent in Epithelial tumors (10.7\%), less differentiated tumours (11.0\%) and younger subjects (13.4\%). Regression tree analysis permitted to individualize a subset of $66 \%$ OC patients with particularly low risk (3.5\%) to carry a BRCA mutation vs a subgroup (24\% of the series), with a probability higher than $17 \%$ to carry a pathogenic mutation. Younger age, OC and Breast Cancer family history were confirmed powerful factors in selecting subgroups of patients with significantly different BRCA mutation probability.
\end{abstract}

Conclusions: Our regression tree-analysis can represent an innovative approach taking into consideration all main clinical pathological information to select OC patients to be candidated for BRCA test.

Keywords: Ovarian cancer, BRCA, Test criteria

\section{Background}

BRCA $1 / 2$ genes play a major role in normal cell DNA repair machinery, participating in the repair of doublestrand breaks by homologous recombination, which, when impaired, is responsible for accumulation of genomic alterations and final genomic instability [1].

The knowledge of these mechanisms permitted to interpret the pathogenic relevance of BRCA1/2 gene mutations in families carrying germline mutations: alterations in these genes confer an higher risk for ovarianfallopian cancer ranging between 39 and $63 \%$ for BRCA 1 and $16-27 \%$ for BRCA2 mutations [2] with respect to

\footnotetext{
* Correspondence: a.paradiso@oncologico.bari.it

${ }^{1}$ Experimental Oncology - Center for Study of Heredo-Familial Tumors,

IRCCS-Istituto Tumori "Giovanni Paolo II", viale Orazio Flacco, 65, 70124 Bari, Italy

${ }^{4}$ Centro Studi Tumori Eredo-Familiari, Istituto Tumori G Paolo II IRCCS National Cancer Research Centre, Via O Flacco, 65, 70124 Bari, Italy Full list of author information is available at the end of the article
}

a mean risk in the overall population of $1-2 \%$. As a consequence, clinical preventive-prophylactic strategies have been proposed (www.nccn.org) for subjects carrying pathogenic mutations in these genes thus making urgent the individualization of appropriate criteria to candidate woman to BRCA1/2 genetic test [3]. The problem of best criteria for genetic counseling enrollment was debated since the last decade generally concluding that presence of familiarity and high risk for BRCA mutation probability calculated by specific softwares [4] should be utilized in routine clinical practice.

More recently, a new and exciting application for BRCA mutation test has been represented by its utilization as Companion Diagnostic Tests (CDX) for drugs Poly-ADP ribose polymerase-Inhibitors (PARP-I). PARP genes encode for proteins playing an essential role in DNA singlestrand breaks repair suggesting that specific PARP-I drugs could be of clinical usefulness first of all by inducing a

(c) The Author(s). 2019 Open Access This article is distributed under the terms of the Creative Commons Attribution 4.0 International License (http://creativecommons.org/licenses/by/4.0/), which permits unrestricted use, distribution, and reproduction in any medium, provided you give appropriate credit to the original author(s) and the source, provide a link to the Creative Commons license, and indicate if changes were made. The Creative Commons Public Domain Dedication waiver (http://creativecommons.org/publicdomain/zero/1.0/) applies to the data made available in this article, unless otherwise stated. 
synthetic lethality effect in cells also carrying a BRCA gene mutations [5]. In 2014, Food and Drug Administration (www.FDA.gov) and European Medicines Agency (www. ema.europa.eu) confirmed the predictive value of BRCA test as CDX for selection of patients to PARP-I treatment after previous response to platinum agents [6, 7]. Considering that several studies reported that an important fraction (about 40\%) of BRCA-mutated ovarian cancer (OC) patients might not have a family history [8] and, furthermore, that BRCA mutational status could provide information also regarding the prognosis and the therapeutic strategy overall, delivering widespread BRCA testing to all OC patients has been proposed by several Authors $[8,9]$, recommended by professional societies $[10-12]$, requested by patients organizations $[13,14]$ and adopted by several authoritative institutions $[15,16]$.

The lack of studies clearly demonstrating the costeffectiveness of widespread BRCA test approach with respect to nowadays standard practice, convinced us of the opportunity to verify if we can better select subgroups of OC patients to be tested for BRCA assay. In order to individualize subsets of cases with higher probability to carry a BRCA1/2 mutation, we applied for the first time multivariate and decision-tree analyses to the largest published database of OC patients, provided of exhaustive clinical and genetic associated information [17].

\section{Methods}

\section{Clinical dataset}

We analysed the database provided by CR-UK, Department of Oncology, University of Cambridge, UK, comprising clinical-pathological and molecular information of 2222 OC women with analyzed BRCA status. Data on germline BRCA analysis by Sanger-sequencing performed in all patients have been already published [17]. All patients included in the database had a previous histological diagnosis of invasive $\mathrm{OC}$ within two casecontrols studies: the population-based SEARCH study (1321 cases) from the United Kingdom, and the Hospital-Mayo clinic study (919 cases) from USA [17].

For 2192 patients the following information were available: Age at diagnosis of OC; Ethnicity (only white woman); Histology (serous, mucinous, endometrioid, clear cell, mixed cell; other specified epithelial OC); cytohistological differentiation (Well, Moderately, Poorly or Undifferentiated Grade, Not assessed); disease stage according to FIGO classification; family history of OC in first degree relative $(\mathrm{OCFh})$; family history of breast cancer in first degree relative (BCFh). The characteristics of the patient series are described in Table 1.

\section{Statistical methods}

The frequencies of all clinical-pathological characteristics already described with respect to presence/absence
Table 1 Description of the clinical-pathological characteristics of the cohort of Ovarian Cancer patients (M\&M for details on categories reported)

\begin{tabular}{|c|c|}
\hline Histology & N (\%) \\
\hline Serous Epithelial & $1312(59,8)$ \\
\hline Mucinous & $143(6,5)$ \\
\hline Endometrioid & $322(14,7)$ \\
\hline Clear Cell & $201(9,2)$ \\
\hline Mixed & $98(4,5)$ \\
\hline Other Epithelial (Brunner) & $79(3,6)$ \\
\hline Undifferentiatied & $5(0,2)$ \\
\hline N/A & $32(1,3)$ \\
\hline \multicolumn{2}{|l|}{ Disease Stage FIGO } \\
\hline 1 & $523(23,9)$ \\
\hline 2 & $249(11,4)$ \\
\hline 3 & $1103(50,3)$ \\
\hline 4 & $25(1,1)$ \\
\hline N/A & $292(13,3)$ \\
\hline \multicolumn{2}{|l|}{ Grade Differentiation } \\
\hline Well & $447(20,4)$ \\
\hline Moderately & $261(11,9)$ \\
\hline Poorly & $1255(57,2)$ \\
\hline Undifferentiated & $15(0,7)$ \\
\hline N/A & $214(9,8)$ \\
\hline \multicolumn{2}{|c|}{ Ovarian Cancer family History } \\
\hline No & $1718(78,4)$ \\
\hline Yes & $107(4,9)$ \\
\hline N/A & $367(16,7)$ \\
\hline \multicolumn{2}{|l|}{ Breast Cancer Family History } \\
\hline No & $1519(69,2)$ \\
\hline Yes & $319(14,5)$ \\
\hline N/A & $354(16,1)$ \\
\hline \multicolumn{2}{|l|}{ BRCA1/2 alterations } \\
\hline Present & $182(8,4)$ \\
\hline Absent & $2010(91,6)$ \\
\hline
\end{tabular}

of BRCA $1 / 2$ mutations were preliminarily analyzed by logistic regression; patients were grouped for further analysis according to histology of the tumour (epithelial vs not-epithelial histology), clinical disease stage (I-II vs III-IV) Differentiation grade (1-2 vs $3-4)$, Age (<50 yrs. vs $>50 \mathrm{yrs}$ ), family history of $\mathrm{OC}$ (OCFh, present vs absent); family history of breast cancer (BCFh, present vs absent).

A multivariate logistic regression analysis with BRCA mutation status as dependent variable was conducted; all the variables included in the model were categorized as described above. 
Finally, a statistical inference analysis was conducted building up a conditional inference tree. Conditional inference trees were performed with the "party" package (version 1.2-2) in the $\mathrm{R}$ system for statistical computing (version 3.3.2, R Development Core Team 2004), both being freely available [18] from CRAN (http:/CRAN.Rproject.org). In detail, for the present study, the tree function has been used to obtain conditional inference regression tree [19]. Such an approach integrates treestructured regression models with conditional inference methods. Rpart algorithm in default mode, as used in the present paper, manages missing values keeping observations even if one or more predictors are lacking.

\section{Results}

Overall, 178 (8.1\%) of OC women showed a germline mutation in BRCA genes, 84 in BRCA 1 and 94 in BRCA2.

The frequency of BRCA mutations resulted higher in Epithelial tumors than in those of not epithelial origin (10.7\% vs $4.2 \%$; $p<0.0001)$; in less vs high differentiated tumours $(11.0 \%$ vs $4.3 \%$; $<0.0001)$ and in younger $<50$ years subjects $(13.4 \%$ vs $6.8 \%$; $<<0.0001)$. However, the highest probability to carry a BRCA mutation has been observed in cases with first degree family history of OC (27.1\%) or BC (17.6\%). Logistic regression analysis (Table 2), confirmed the significant difference between women with vs without OCFh (Odds ratio, OR: 4.9; 95\% CI 3.04-7.73) and for women with vs without BCFh (OR: 3.19; 95\% CI 2.22-4.53).

The multivariate regression analysis (Table 3), confirmed the predictive role for presence of BRCA mutation, of OCFh (OR 3.91;95\%CI 2.1-7.04) and of BCFh (OR 3.75 ; 2.46-5.67); conversely, older $>50$ yrs. women

Table 2 Logistic Regression Analysis with BRCA mutation status as dependent variable in different clinical-pathological subsets of cases

\begin{tabular}{lll}
\hline Logistic Regression & & \\
\hline \multicolumn{1}{l}{ Histology } & Odds ratio $(95 \% \mathrm{Cl})$ & $p$-value \\
$\quad$ Non Epithelial vs Epithelial & $0.37(0.25 \div 0.53)$ & $<0.0001$ \\
$\begin{array}{l}\text { Stage } \\
\text { II-III vs I }\end{array}$ & $2.69(1.7 \div 4.49)$ & $<0.0001$ \\
$\begin{array}{l}\text { CytoHistological Differentiation Grade } \\
\text { III vs I-II }\end{array}$ & $2.7(1.83 \div 4.1)$ & $<0.0001$ \\
Age & $0.47(0.34 \div 0.65)$ & $<0.0001$ \\
$>50$ years vs $<50$ years & & \\
Ovarian Cancer history & $4.9(3.04 \div 7.73)$ & $<0.0001$ \\
$\quad$ 1st Degree Affected vs Not & & $<0.0001$ \\
Breast Cancer history & $3.19(2.22 \div 4.53)$ & \\
1st Degree Affected vs Not & & \\
\hline
\end{tabular}

Table 3 Multivariate Logistic Regression Analysis with BRCA mutation status as dependent variable in different clinicalpathological subsets of cases

\begin{tabular}{|c|c|c|}
\hline \multicolumn{3}{|l|}{ Multivariate Logistic Regression } \\
\hline & Odds ratio $(95 \% \mathrm{Cl})$ & $p$-value \\
\hline \multicolumn{3}{|l|}{ Histology } \\
\hline Non Epithelial vs Epithelial & $0.48(0.26 \div 0.83)$ & 0.012 \\
\hline \multicolumn{3}{|l|}{ Stage } \\
\hline||$-|| \mid$ vs $\mid$ & $1.43(0.76 \div 2.82)$ & 0.28 \\
\hline \multicolumn{3}{|c|}{ CytoHistological Differentiation Grade } \\
\hline III vs I-II & $2.34(1.34 \div 4.3)$ & 0.003 \\
\hline \multicolumn{3}{|l|}{ Age } \\
\hline$>50$ years vs $<50$ years & $0.27(0.18 \div 0.43)$ & $<0.0001$ \\
\hline \multicolumn{3}{|l|}{ Ovarian Cancer history } \\
\hline 1st Degree Affected vs Not & $3.91(2.1 \div 7.04)$ & $<0.0001$ \\
\hline \multicolumn{3}{|l|}{ Breast Cancer history } \\
\hline 1st Degree Affected vs Not & $3.75(2.46 \div 5.67)$ & $<0.0001$ \\
\hline
\end{tabular}

showed a significantly lower probability (OR 0.27; 95\%CI: $0.18-0.43)$ to be BRCA1/2 mutation carriers.

In order to check for the possibility to better select subgroups of women with different probability frequency of BRCA mutations, we applied a regression tree analysis to a large series of clinical and molecular well characterized OC patients (Song, 2014). We highlighted a decisional tree with nodes indicating subgroups of patients significantly different for probability to carry a BRCA mutation (Fig. 1) with 8 terminal subgroups of $\mathrm{OC}$ women with a probability to carry a mutation ranging from 1 to $40 \%$. This probability resulted particularly high (40\%) in OC patients younger than 46 yrs., with BCFh and OCFh (NODE 6); in OC patients with OCFh+ (29\%) (NODE 8 ). Conversely, that probability resulted: very limited ( $<1 \%$ of BRCA mutation frequency) in patients with negative OCFh and BCFh/Disease Stage I-II/ Differentiation Grade I-II (NODE 1); in patients without OCFh and BCFh/Disease Stage III/ Younger Age/ Grade I-II (3\% of overall series of patients with BRCA mutation frequency $<3 \%$ ) (NODE 3$)$.

\section{Discussion}

Previous papers highlighted the great variability in terms of percentage of BRCA alteration in series of OC patients reported by different Authors. de-Jong [9] reported an overall probability of presence of germline BRCA $1 / 2$ mutations above $10 \%$ in 6218 women with epithelial OC confirming three referral criteria to candidate $\mathrm{OC}$ women to genetic counseling: age of onset, family history of $\mathrm{BC}$ and/or $\mathrm{OC}$ and histology. However, he also stressed that categories not fulfilling these selection criteria still have a substantial probability of carrying a germline BRCA mutation concluding that "testing 


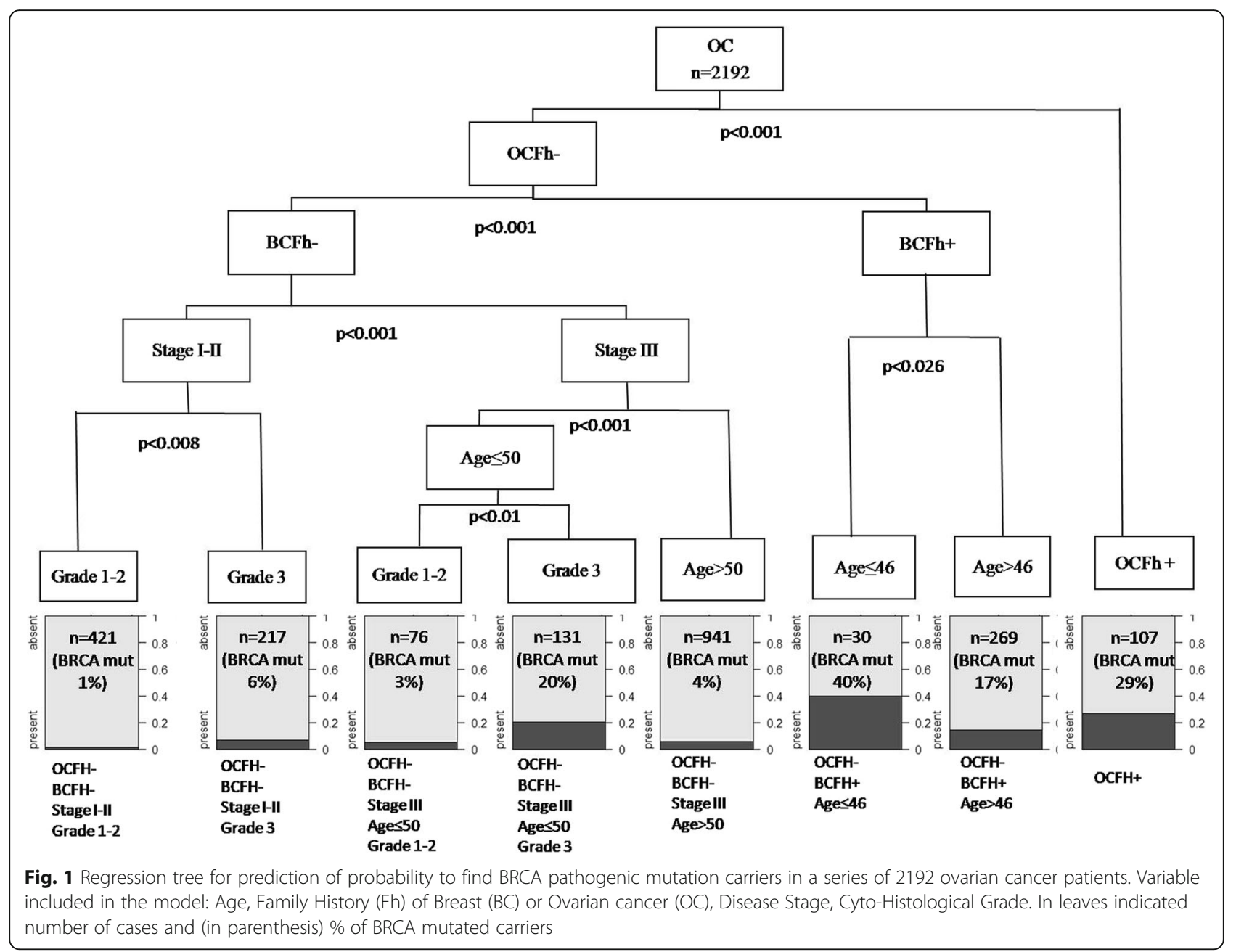

should be offered regardless of those characteristics otherwise an important part of germline BRCA1/2 mutation carriers could be missed" [9]. This position was supported by several authors [8]. Furthermore, the recent updated 3.2019 NCCN guidelines do no longer consider the possibility to utilize genetic risk models (like BRCAPRO) for a better selection of candidates to BRCA test (https://www.nccn.org/professionals/physic ian_gls/pdf/genetics_screening.pdf).

In the present series we showed by univariate and multivariate analyses that BRCA mutation rate is strongly associated with epithelial histology, low citohistological tumour differentiation and, first of all, with OC or BC family history in first degree relatives (Tables 2 and 3). Several Authors confirmed that the epithelial cancer histology is associated to BRCA mutations. Indeed, Alsop [20] reported a germline BRCA1/2 mutation in $14 \%$ of 1001 women with non-mucinous epithelial ovarian cancer (EOC); Zhong [21] pointed out the presence of $17 \%$ of BRCA mutations by reviewing a series of 9588 epithelial EOC. In our series of patients, we demonstrated $10.7 \%$ percentage of BRCA mutation in epithelial origin $\mathrm{OC}$ women; however, as already reported [17], gene deletions and duplications in this series were not analyzed, even if, Kwong [22] demonstrated that large deletions or duplications in BRCA1/2 genes, accounts for $0.7 \%$ of all BRCA pathogenic alterations, only.

The most intriguing results of the present paper, came from regression tree analysis which showed that there are subgroups of patients, characterized by a combination of clinical-pathological factors and an enormous difference in BRCA mutation frequencies. For first our original analysis confirms the strong impact that OC and/or BC family history has in determining the probability to carry a BRCA mutation and this, irrespective to age for OC.

We further demonstrated that there is a subgroup of about $20 \%$ of all OC patients (without family history, early disease stage, well differentiated) with $<1 \%$ of BRCA mutation rate; moreover, a subgroup of $46 \%$ of patients, included in Nodes 3 and 5 of the Regression- 
Tree (Fig. 1 ) showed a $<3.8 \%$ probability only to carry a BRCA mutation. Conversely, this probability resulted particularly high $(>40 \%)$ in young women with BCFh (Node 6) or with OCFh (>29\%) (Node8). This is the first time that the concept of hierarchy and of multifactor risk is associated to BRCA mutation rate in a large series of $\mathrm{OC}$ women. In fact, our hierarchical approach permitted to individualize patients belonging to Nodes 1-3-5 of the Tree, representing a subset of $66 \%$ of all OC patients, with particularly low risk to carry a BRCA mutation vs a subgroup of $\mathrm{OC}$ women, representing the $24 \%$ of all the series, with a probability to carry a pathogenic mutation always over $17 \%$.

The main conclusion from these data is that the probability to find a BRCA mutation varies greatly in different clinical subgroups leading to the hypothesis that testing for BRCA mutations in OC patients could be better addressed within each specific clinical scenario and according to better defined cost-effective programs.

The question of how to manage, in a cost-effective way, BRCA tests for OC patients has generated a wide debate. D'Andrea [23], after a systematic review on economic evaluations on BRCA genetic testing programs, concluded that there is no evidence of cost-effectiveness for BRCA screening to all newly diagnosed cases of OC cancer even though followed by cascade testing of relatives. Kwon [24], estimating the cost-effectiveness of BRCA mutation testing in USA and the down stream benefits for first degree relatives, confirmed that the benefit concerned only OC women, with a personal history of breast and/or OC. Slade [25] stressed the need for adherence to NICE elegibility criteria requiring a BRCAPRO risk $>10 \%$ to reach a useful cost-effectiveness ratio. Eccleston [26], in a study conducted in UK, reached different conclusions reporting that implementing routine BRCA testing in women with $\mathrm{OC}$ would be cost-effective but only if compared with no testing to all patients policy.

We can therefore affirm that there is no evidence of a clear cost-efficacy benefit for widespread genetic test to all OC patients when compared to testing selected subgroups of patients only. On the other hand, we have to stress that there is no demonstration that our regression tree model can represent an alternative more costeffective approach with respect to standard practice. A study to directly compare the performances of our innovative approach with respect to BRCAPRO is ongoing.

However, there is general agreement about the fact that new technological approach (i.e. massive sequencing) will dramatically lower costs and then the costefficacy equilibrium for wide BRCA test utilization policies [22-24].

An important point supporting the implementation of widespread testing strategies has been the utilization of such test as predictive biomarker for PARP-I stated from FDA and EMA, and, more in general, for an optimal therapeutic strategy design for $\mathrm{OC}$ women $[8,27]$. In particular, PARP-I utilized in OC women carrying a BRCA mutation as maintenance therapy, has proven to dramatically improve the outcome of these patients [28].

However, regarding these points, alternative views have to be discussed. In 2017, the FDA (www.accessdata. fda.gov) approved two PARP inhibitors, olaparib and niraparib, as maintenance treatment for women with OC who respond to induction platinum-based chemotherapy, regardless of their BRCA-mutation status [28, 29]; recent findings from the phase III ARIEL3 trial of rucaparib corroborate the genotype agnostic benefit of PARP inhibition [30]. Moreover, Tan [31] supported the idea that the delivery of BRCA test as predictive to response to other common drugs (platinum derivatives, trabectedin) utilized for OC patients has to be still considered as an experimental approach. It seems we can conclude that, to date, to know the BRCA test to consider PARP-I utilization and for a better planning a complete therapeutic strategy for OC patients, cannot be simply supported.

Interestingly, the ARIELIII trial scientists stressed the urgent need to a deeper study of homologous recombination repair deficiency (HRD) in patients candidate to PARP-I treatment, also considering the potential harmful effect of false negative BRCA results leading to false patient's reassurance and to appropriate care neglection [30].

\section{Conclusions}

In conclusion, while some countries are already considering national wide roadmaps to facilitate and improve BRCA genetic testing rates [32], we suggest that there is no evidence that delivering a widespread BRCA testing for $\mathrm{OC}$ patients is cost effective with respect to standard practice for preventive and therapeutic purposes. The possibility to better select candidates to the test is a feasible approach and, from this perspective, our regression tree analysis could represent a reasonable practical approach. A better selection of patients to be tested together with new predictive biomarkers looking, in a deeper and wider way, at HRD characteristics of OC patients are urgently needed.

\section{Abbreviations \\ BC: Breast cancer; BCFh: Breast Cancer in first degree relative; \\ CDX: Companion Diagnostic Text; HRD: homologous recombination DNA repair deficiency; OC: Ovarian Cancer; OCFh: Ovarian Cancer in first degree relative; PARP-I: Poly-ADP ribose polymerase-Inhibitors}

\section{Acknowledgments}

We thank P Pharoah and L Song fron UK-CRUK for the availability to utilize their OC databases for the present study.

\section{Authors' contributions}

AVP planned the study, took care of contacts with P. Pharoah and H. Song from CR-UK, Department of Oncology, University of Cambridge, UK and 
wrote the manuscript; SD performed statistical analyses; MD and MP organized the $\mathrm{db}$ and performed univariate analyses; ST and IBN contributed to manuscript writing and revision. All authors read and approved the final manuscript.

\section{Funding}

The study has been funded by Ministry of Health of Italian Gouvernment. Funds for Institutional Research, 2016.

\section{Availability of data and materials}

All data materials and analyses are available upon request and only after approval of UK-CRUK Referents

\section{Ethics approval and consent to participate}

All study subjects provided written, informed consent to participate in the study approved by Ethics Committee (Song H et al., 2014). The present Project was approved by IAB of IRCCS-Istituto tumori G Paolo II Bari, Italy.

\section{Consent for publication}

Not Applicable.

\section{Competing interests}

The authors declare that they have no competing interest.

\section{Author details}

'Experimental Oncology - Center for Study of Heredo-Familial Tumors, IRCCS-Istituto Tumori "Giovanni Paolo II", viale Orazio Flacco, 65, 70124 Bari, Italy. ${ }^{2}$ Molecular and Pharmacogenetics Diagnostic Laboratory, IRCCS-Istituto Tumori "Giovanni Paolo II", viale Orazio Flacco, 65, 70124 Bari, Italy. ${ }^{3}$ Department of Functional Genomics and Experimental Pathology, The Oncology Institute "Prof. Dr. Ion Chiricuta", University of Medicine and Pharmacy luliu Hatieganu- Center for Functional Genomics and Center for Advanced Medicine Medfuture, Republicii 34th street; Marinescu 23, Pasteur 4-6, 400015 Cluj-Napoca, Romania. ${ }^{4}$ Centro Studi Tumori Eredo-Familiari, Istituto Tumori G Paolo II IRCCS National Cancer Research Centre, Via O Flacco, 65, 70124 Bari, Italy

\section{Received: 3 September 2018 Accepted: 12 June 2019}

\section{Published online: 28 June 2019}

\section{References}

1. Auguste A, Leary A. Abnormalities of DNA repair and gynecological cancers. Bull Cancer. 2017;104(11):971-980.

2. Mavaddat N, Peock S, Frost D, Ellis S, Platte R, Fineberg E, et al. Cancer risks for BRCA1 and BRCA2 mutation carriers: results from prospective analysis of EMBRACE. J Natl Cancer Inst. 2013;105(11):812-22.

3. https://www.nccn.org/professionals/physician_gls/pdf/genetics_screening. pdf

4. Profato JL, Arun BK. Genetic risk assessment for breast and gynecological malignancies. Curr Opin Obstet Gynecol. 2015;27(1):1-5.

5. Lord CJ, Ashworth A. Mechanisms of resistance to therapies targeting BRCAmutant cancers. Nat Med. 2013;19(11):1381-8.

6. https://www.fda.gov/Drugs/InformationOnDrugs/ApprovedDrugs/ ucm533891.htm

7. http://www.ema.europa.eu/ema/index.jsp?curl=pages/medicines/human/ medicines/003726/human_med_001831.jsp\&mid=WC0b01ac058001d124.

8. George A, Kaye S, Banerjee S. Delivering widespread BRCA testing and PARP inhibition to patients with ovarian cancer. Nat Rev Clin Oncol. 2017;14(5):284-96.

9. Arts-de Jong M, de Bock GH, van Asperen CJ, Mourits MJ, de Hullu JA, Kets CM. Germline BRCA1/2 mutation testing is indicated in every patient with epithelial ovarian cancer: a systematic review. Eur J Cancer. 2016:61:137-45.

10. Marth C, Hubalek M, Petru E, Polterauer S, Reinthaller A, Schauer C, et al. AGO Austria recommendations for genetic testing of patients with ovarian cancer. Wien Klin Wochenschr. 2015;127(15-16):652-4.

11. Pinto C, Bella MA, Capoluongo E, Carrera P, Clemente C, Colombo N, et al. Recommendations for the implementation of BRCA testing in the care and treatment pathways of ovarian cancer patients. Future Oncol. 2016;12(18):2071-5.
12. Vergote I, Banerjee S, Gerdes AM, van Asperen C, Marth C, Vaz F, et al. Current perspectives on recommendations for BRCA genetic testing in ovarian cancer patients. Eur J Cancer. 2016;69:127-34.

13. Arie S. Routine testing for women with ovarian cancer. BMJ. 2014;348:g1200.

14. Wise J. Charity calls for routine BRCA testing of ovarian cancer patients. BMJ. 2013;347:f5664.

15. Levine DA, Karlan BY, Strauss JF. Evolving approaches in research and Care for Ovarian Cancers: a report from the National Academies of sciences, engineering, and medicine. JAMA. 2016;315(18):1943-4.

16. NHS. Developing Testing Criteria for Familial Breast and Ovarian Cancer: Incorporating NICE Guidelines. UK Genetic Testing Network. Meeting report from Chandos House, London (2014). Available at Http://kgtn.nhs.uk/ fileadmin/uploads/ukgtn/Documents/Resources/Library/Reports_Guidelines/ UKGTN\%20breast\%20cancer\%20Final\%20161014.pdf.

17. Song $H_{1}$ Cicek MS, Dicks E, Harrington P, Ramus SJ, Cunningham JM, et al. The contribution of deleterious germline mutations in BRCA1, BRCA2 and the mismatch repair genes to ovarian cancer in the population. Hum Mol Genet. 2014:23(17):4703-9.

18. https://CRAN.R-project.org

19. Hothorn T, Hornik K, Zeileis A. Unbiased recursive partitioning: a conditional inference framework. J Comput Graph Stat. 2006;15(3):651-74.

20. Alsop K, Fereday S, Meldrum C, deFazio A, Emmanuel C, George J, et al. BRCA mutation frequency and patterns of treatment response in BRCA mutation-positive women with ovarian cancer: a report from the Australian Ovarian Cancer Study Group. J Clin Oncol. 2012;30(21):2654-63.

21. Zhong Q, Peng HL, Zhao X, Zhang L, Hwang WT. Effects of BRCA1- BRCA2related mutations on ovarian and breast cancer survival: a meta-analysis. Clin Cancer Res. 2015;21:211-20.

22. Kwong A, Chen J, Shin VY, Ho JC, Law FB, Au CH, et al. The importance of analysis of long-range rearrangement of BRCA1 and BRCA2 in genetic diagnosis of familial breast cancer. Cancer Genet. 2015;208(9):448-54

23. D'Andrea E, Marzuillo C, De Vito C, Di Marco M, Pitini E, Vacchio MR, et al. Which BRCA genetic testing programs are ready for implementation in health care? A systematic review of economic evaluations. Genet Med. 2016:18(12):1171-80

24. Kwon JS, Gutierrez-Barrera AM, Young D, Sun CC, Daniels MS, Lu KH, et al. Expanding the criteria for BRCA mutation testing in breast cancer survivors. J Clin Oncol. 2010;28(27):4214-20.

25. Slade I, Hanson H, George A, Kohut K, Strydom A, Wordsworth S, et al. A cost analysis of cancer genetic service model in the UK. J Community Genet. 2016;7(3):185-94

26. Eccleston A, Bentley A, Strydom A, Vereecken W, George A, Rahman N. A cost-effectiveness evaluation of germline BRCA1 and BRCA2 testing in UK women with ovarian Cancer. Value Health. 2017:20(4):567-76.

27. Moore K, Colombo N, Scambia G, Kim BG, Oaknin A, Friedlander M, et al. Maintenance Olaparib in patients with newly diagnosed advanced ovarian Cancer. N Engl J Med. 2018;379(26):2495-505.

28. https://www.accessdata.fda.gov/drugsatfda docs/label/2017/208447/bl.pdf

29. Stewart J, George A, Banerjee S. Olaparib for the treatment of relapsed ovarian cancer with a BRCA1/2 mutation. Expert Rev Anticancer Ther. 2018; 18(10):947-58

30. Coleman RL, Oza AM, Lorusso D, Aghajanian C, Oaknin A, Dean A, et al. Rucaparib maintenance treatment for recurrent ovarian carcinoma after response to platinum therapy (ARIEL3): a randomised, double-blind, placebo-controlled, phase 3 trial. Lancet. 2017:390(10106):1949-61.

31. Tan DSP, Kaye SB. Chemotherapy for patients with BRCA1/2- mutated ovarian cancer: same or different? ASCO Educational Book; 2015. p. 114-21. Asco.org/edbook

32. McCuaig JM, Stockley TL, Shaw P, Fung-Kee-Fung M, Altman AD, Bentley J, et al. BRCA to Community of Practice. Evolution of genetic assessment for BRCA-associated gynaecologic malignancies: a canadian multisociety roadmap. J Med Genet. 2018;55(9):571-7.

\section{Publisher's Note}

Springer Nature remains neutral with regard to jurisdictional claims in published maps and institutional affiliations. 